\title{
Compartment Syndrome Following Robotic Pelvic Urological Surgery: Diagnosis and Management
}

\author{
Narin Suleyman ${ }^{1}$, Ahilan Pathmanathan ${ }^{2}$,Todd Kanzara ${ }^{1}$, Tim Lane ${ }^{1}$, Greg Boustead ${ }^{1}$, Jim Adshead ${ }^{1}$, Gowrie \\ Mohan $\mathbf{S}^{2}$, Nikhil Vasdev ${ }^{1^{*}}$ \\ ${ }^{1}$ Hertfordshire and South Bedfordshire Robotic Urological Cancer Centre, Department of Urology, Lister Hospital, Stevenage \\ ${ }^{2}$ Department of Anesthetics, Lister Hospital, Stevenage
}

*Corresponding author: Nikhil Vasdev, Consultant Urological and Robotic Surgeon, Lister Hospital, Stevenage, SG14AB, United Kingdom; E-mail: nikhilvasdev@doctors.org.uk
Citation: N. Vasdev., et al. Compartment Syndrome Following Robotic Pelvic Urological Surgery: Diagnosis and Management (2015) J Anesth Surg 2(1): 26-29.

\section{Received date: January 20, 2015 \\ Accepted date: January 22, 2015 \\ Published date: January 29, 2015}

\section{Introduction}

Compartment syndrome is the elevation of intra compartmental pressure to a level that impairs circulation by exceeding perfusion pressure ${ }^{[1]}$. The importance in understanding this condition is in it's high-risk nature, diagnostic challenge and the urgency of intervention that is required with an acute presentation $^{[1]}$. We present the commonest forms of compartment syndrome (lower limb and abdominal) which can present both intra and post-operatively during robotic pelvic urological surgery.

\section{Limb (Upper and Lower) Compartment Syndrome}

Acute compartment syndrome of the limbs is most often associated with acute injury to the lower leg, but can develop throughout the upper and lower limbs, the buttocks and paraspinal muscles ${ }^{[1]}$. Fracture continues to be the commonest primary cause, specifically that of the Tibial shaft ${ }^{[2]}$. In addition, vascular injury, burns, crush injury ${ }^{[1]}$ injection injury, reperfusion injury, bleeding disorders, infection, constrictive dressings, casts and extravasation of drugs have all been described as a cause of acute compartment syndrome ${ }^{[3]}$. The incidence is 3.1 per 100000 population $^{[2]}$. Fracture-related compartment syndrome is more common in younger males with fewer co-morbidities, compared to the atraumatic cohort, who are more at risk of posterior compartment syndrome ${ }^{[2]}$. The risk of atraumatic compartment syndrome is greater in patients with obesity and peripheral vascular disease ${ }^{[4]}$. Patients undergoing surgery in the lithotomy position are at an increased risk, which increases further with a procedure time of over four hours and a less experienced surgeon ${ }^{[4]}$.

\section{Pathophysiology}

Compartment syndrome can occur in any area of the body with little capacity for tissue expansion ${ }^{[3]}$ with a similar mechanism of injury in each location. Increasing its content or decreasing the size of the compartment can raise compartment pressure. This triggers a vicious cycle whereby increased venous pressure causes an increased capillary pressure and the increase in exudate increases the content of the compartment further. De- creasing tissue perfusion by reducing arteriolar pressure eventually increases capillary permeability and causes a further increase in exudate. Muscle and nerve necrosis follows, causing the earliest clinical signs of compartment syndrome. Figure 1

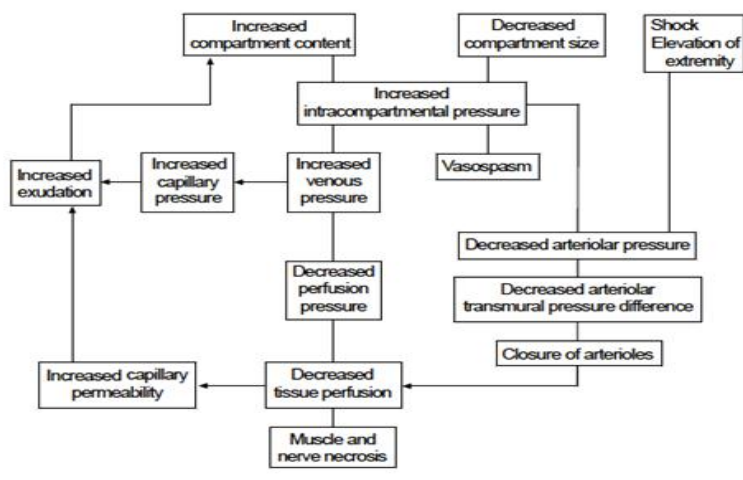

Figure 1: The Pathophysiology of Compartment Syndrome

The Lower Limb contains four osseo fascial compartments by interosseous membrane of the leg, transverse intermuscular septum and anterior intermuscular (crural) septum (Figure 2).

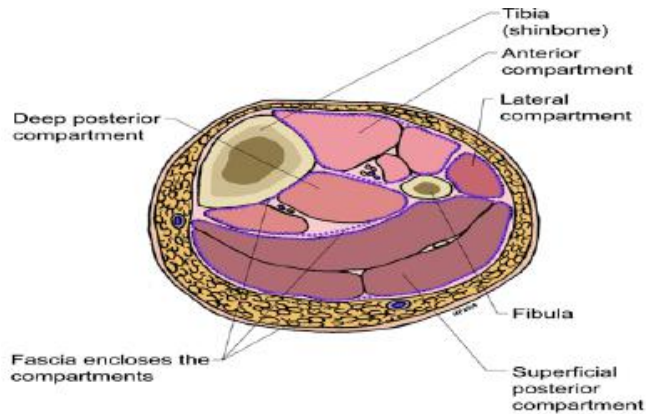

Figure 2: Anterior compartment muscular tibialis anterior extensor hallucislongus, extensor digitorumlongus peroneustertius neurovascular deepperoneal nerve anteriortibial vessels Lateral compartment muscular peroneuslongus peroneusbrevis neurovascular superficialperoneal nerve Superficial posterior compartment muscular gastrocnemius plantaris soleus neurovascular sural nerve Deep posterior compartment muscular tibialis posterior flexorhallucislongus flexordigitorumlongus popliteus neurovascular tibial nerve posteriortibial vessels.

Copy rights: (C2015 N. Vasdev. This is an Open access article distributed under the terms of Creative Commons Attribution 4.0 International License. 


\section{Diagnosis}

Diagnosis is delayed in those with an atraumatic cause $^{[5]}$, patients whose mental status prevents communication of pain ${ }^{[1]}$ and those with long-acting nerve blocks, which can mask the early signs of compartment syndrome ${ }^{[6]}$.

\section{Clinical}

Compartment syndrome has typically been a clinical diagnosis, comprising of the presence of the six "P's". These are pain, on passive stretch of the muscles in question ${ }^{[3]}$, out of proportion to the injury and increasing analgesia requirements, parasthesia as an early feature due to ischaemia of peripheral nerves, paralysis, pallor, pulselessness, and poikothermia ${ }^{[1]}$. The latter three of these are late signs, by which time ischaemic changes may be irreversible ${ }^{[3]}$.

\section{Pressure Measurement}

Measurement of intra-compartmental pressures may not be necessary if there is a high index of clinical suspicion. However, when doubt remains, a transducer connected to a catheter is introduced under aseptic conditions into the compartment, within $5 \mathrm{~cm}$ of the zone of injury ${ }^{[3]}$. There is a case for continuous intra-compartmental pressure monitoring in all patients at risk of compartment syndrome, in order to detect it before the onset of clinical signs and reducing time to definitive treatment and decreasing complication rate $\mathrm{e}^{[7]}$.

Healthy muscle has an intra-compartmental pressure of around $10 \mathrm{mmHg}^{[8]}$. A pressure of $30 \mathrm{mmHg}$ is thought to be the level at which microcirculation is compromised as muscle blood flow cannot be maintained and the fascia is maximally stretched ${ }^{[9]}$. This level is different to the pressure at which irreversible damage is done, as this varies according to the perfusion pressure and whether a patient's blood pressure is able to maintain adequate tissue perfusion ${ }^{[10]}$. The difference between the diastolic blood pressure and the intra-compartmental pressure is known as the 'delta pressure ${ }^{[3]}$. A delta pressure of $30 \mathrm{mmHg}$ or below is widely accepted as the point at which treatment should be initiated ${ }^{[11,2]}$.

\section{Anesthetic Considerations}

As already mentioned, prolonged positioning in lithotomy poses an increased risk of compartment syndrome. The reasons for this are thought to be multifactorial.

Firstly, arterial perfusion of the lower limb is reduced when the legs are elevated above the level of the heart. This may be further impaired by Trendelenberg positioning ${ }^{[4]}$. Any hypotension associated with general anaesthesia and/or central neuraxial blockade may further exacerbate this. Furthermore, in the presence of peripheral vascular disease, the onset of microvascular ischaemia may be significantly accelerated and therefore a high degree of vigilance should be maintained ${ }^{[13]}$. Secondly, venous stasis may compromise blood flow to the lower limb. This may arise through kinking of the veins in the groin or from external pressure exerted by stirrups ${ }^{[13]}$. Finally, an increased compartmental pressure related to the weight of the limb may also reduce flow and thus patients with a high body mass index are at higher risk ${ }^{[22]}$. Similarly, antiembolism stockings and the repeated cycling of pneumatic compression calf pumpsare also implicated with a reduction in flow ${ }^{[13]}$.
The pain associated with microvascular ischaemia has a low sensitivity and positive predictive value in diagnosing acute compartment syndrome ${ }^{[14]}$. Moreover, the administration of any form of analgesia has been implicated in delayed recognition of symptoms ${ }^{[14]}$. Despite this controversy, it is considered inhumane to withhold analgesia for this purpose. The evidence that regional and patient controlled analgesia delays diagnosis is also unconvincing ${ }^{[6,14]}$. On the contrary, it may actually facilitate diagnosis with breakthrough pain in the presence of a good working block ${ }^{[14]}$. As such, the use of low dose local anesthetics in central neuraxial blockade for analgesic purposes is considered acceptable. On the other hand, a dense sensory and motor block is not to be recommended.

\section{Treatment}

\section{Risk Management}

Paying attention to details such as intraoperative positioning, anaesthetic choice, stocking and splint placement can decrease the risk of acute compartment syndrome ${ }^{[1]}$. In this regard, measures that may be employed include limiting the height of the legs above the heart ${ }^{[15]}$, maintaining an adequate blood pressure for the duration of surgery and improving padding around the legs to avoid pressure on the calves ${ }^{[15]}$. If surgery is prolonged, placing the legs supine after 2 hours is recommended, in addition to minimizing the overall duration of surgery. Staff vigilance, education and training also contribute to minimizing the risk.

The use of pulse oximetry in the diagnosisis not reliable as pulses may still be palpable in the early stages of acute compartment syndrome ${ }^{[16]}$. Another non-invasive monitoring method includes Near-Infrared spectroscopy (NIRS) which may be more sensitive, though this is expensive and only measures to a limited depth ${ }^{[16]}$. In cases where the diagnosis is unclear, creatine phosphokinase $(\mathrm{CK})$ released during muscle necrosismay be helpful ${ }^{[16]}$.

\section{Immediate Measures}

Immediate management involves the removal of external compressive forces and releasing any casts or dressings. The limb should be kept at the level of the heart for optimal arterial flow $^{[3]}$. There is a threat to life as well as limb and potential renal failure must be anticipated and aggressively countered with intravenous fluids and supplemental oxygen. Early assessment of hypovolaemia, metabolic acidosis and myoglobinaemia must be carried out in conjunction with regular blood biochemistry and urinalysis. A normal cardiac output and blood pressure will maintain perfusion and avoid compounding any existing tissue injury ${ }^{[17]}$.

\section{Surgical Treatment}

If clinical features do not improve with simple measures, emergency definitive surgical fasciotomy is indicated within six hours to prevent irreversible myoneural damage ${ }^{[3]}$. The aims of fasciotomy are to achieve an adequate and extensile incision, complete release of all compartments involved whilst preserving vital structures ${ }^{[3]}$. Thorough debridement is part of the procedure and a second look with further debridement is usually required 48-2 hours later ${ }^{[3]}$. Orthopaedic, vascular and plastic surgery input may be required to deal with the associated 
injuries. Primary amputation may be indicated if diagnosis has been delayed in a case of significant trauma where muscle function is absent ${ }^{[3]}$. Fasciotomy after eight hours is controversial and the potential benefit is outweighed by the increased risk of infection, delay to amputation and multi-organ failure ${ }^{[18]}$. Figure 3
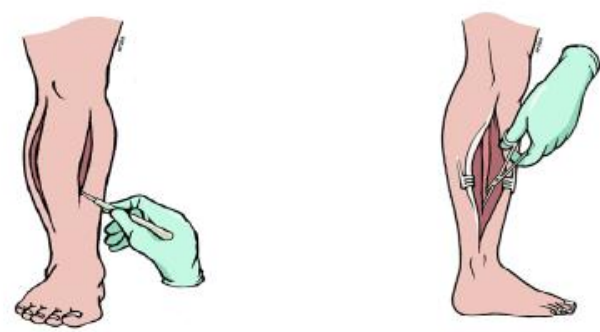

Figure 3: Fasciotomy Incision

\section{Wound Management}

Wound management remains a controversial subject with a variation in practice. Most leave the wound open with delayed primary closure or skin grafting at day 7-10 after complete resolution of the compartment syndrome ${ }^{[3]}$. In the interim, coverage is achieved with simple absorbant dressings, semi-permeable membranes, a 'bootlace' pattern of vessel loops or negative pressure dressings ${ }^{[3]}$.

\section{Complications}

Delayed diagnosis increases the risk of failed treatment, poor outcomes, additional operations, the possibility of amputation, loss of motion, increased expenses and legal ramifications $^{[1]}$.

\section{Systemic}

Post-operatively, pain is a major feature and regular analgesia is required ${ }^{[3]}$. Vigilance regarding rhabdomyolysis and acute renal failure is required and adequate urine output should be maintained with intravenous fluids ${ }^{[3]}$. Renal replacement therapy may be required to avoid intractable hyperkalaemia and subsequent death ${ }^{[4]}$. Forced-alkaline diuresis with mannitol may be beneficial in preventing the acute renal failure secondary to rhabdomyolysis ${ }^{[16]}$.

\section{Wound Complications}

There are multiple complications associated with fasciotomy, in order of decreasing frequency;

Altered sensation within the margins of the wound (77\%)

Dry, scaly skin (40\%)

Pruritus (33\%)

Discolored wounds (30\%)

Swollen limbs $(25 \%)$

Tethered scars $(26 \%)$

Recurrent ulceration (13\%)

Muscle herniation (13\%)

Pain related to the wound (10\%)

Tethered tendons $(7 \%)$

Chronic venous insufficiency due to impaired calf muscle pumps $^{[19]}$

\section{Legal}

Acute compartment syndrome is a common source of litigation and most frequently, in cases where compartment pressures were not measured, there was a malpositioning of the compartment monitor or another equipment error ${ }^{[3]}$.

\section{Abdominal Compartment Syndrome}

Abdominal compartment syndrome is a sustained intra-abdominal pressure greater than $20 \mathrm{mmHg}$ with the presence of an attributable organ failure ${ }^{[20]}$. Again, a successful outcome is reliant on early and accurate diagnosis and timely definitive treatment $t^{[21]}$.

\section{Causes and Diagnosis}

The aggressive fluid resuscitation associated with goal directed therapies have been linked with the undesired consequence of abdominal compartment syndrome ${ }^{[21]}$, occurring in $4.2 \%$ of patients requiring critical care ${ }^{[19]}$. The groups most at risk are the post-operative patients who have experienced near exsanguination, patients who have undergone large volume fluid resuscitation for severe sepsis or thermal injury, and general surgical patients who have required large volume fluid replacement for an abdominal pathology ${ }^{[21]}$. The gold standard for monitoring intra-abdominal pressure is the intravesicular technique; using a pressure transducer attached to an indwelling urinary catheter and a syringe to infuse fluid ${ }^{[22]}$.

\section{Pathophysiology}

Many organ systems are vulnerable to the effects of a raised intra-abdominal pressure. The cardiac system is affected due to decreased venous return ${ }^{[21]}$ and the respiratory system is affected by the functional restriction of diaphragmatic movement and pulmonary expansion, with subsequent hypoxia ${ }^{[23]}$. Renal dysfunction is due to extrinsic renal vein and inferior vena cava compression, causing reduced glomerular filtration and subsequent water conservation ${ }^{[24]}$, in addition to the rhabdomyolysis caused by muscle crush injury ${ }^{[24]}$. In addition reperfusion of the central nervous system, liver and gastrointestinal tract can cause visceral oedema ${ }^{[21]}$.

\section{Treatment}

Medical and surgical treatments of abdominal compartment syndrome need not be mutually exclusive. The main aim of therapy is to evacuate intraluminal contents and intra-abdominal space lesions, improve abdominal wall compliance and to optimize fluids and perfusion ${ }^{[20]}$. Therefore NG tubes, rectal tubes and enemas are used in conjunction with percutaneous drainage and surgical evacuation of abdominal contents ${ }^{[20]}$. If laparostomy is performed, at least one reexploration is required prior to definitive closure ${ }^{[25]}$. There are many wound management options if unable to close, including techniques to stretch fascia and temporary mesh followed by a split thickness graft ${ }^{[21]}$. Abdominal wall reconstruction usually follows 6 to 12 months after the last operation, after inflammation has subsided ${ }^{[21]}$.

\section{References}

1. Garner, M.R., Taylor, S.A., Gausden, E., et al. Compartment syndrome: diagnosis, management, and unique concerns in the twenty-first century. (2014) HSSJ 10(2): 143-152

2. McQueen, M.M., Gaston, P., Court Brown, C.M. Acute compartment 
syndrome. Who is at risk? (2000) J Bone Joint Surg Br 82(2): 200-203

3. Donaldson, J., Haddad, B., Khan, W.S. The pathophysiology, diagnosis and current management of acute compartment syndrome. (2014) Open Orthop J 8:185-193.

4. Pridgeon. S., Bishop, C.V., Adshead, J. Lower limb compartment syndrome as a complication of robot-assisted radical prostatectomy: the UK experience. (2013) BJU Int 112(4): 485-488.

5. Hope, M.J., McQueen, M.M. Acute compartment syndrome in the absence of fracture. (2004) J Orthop Trauma 18(4): 220-224.

6. Mar, G.J., Barrington, M.J., McGuirk, B.R. Acute compartment syndrome of the lower limb and the effect of postoperative analgesia on diagnosis. (2009) Br J Anaesth 102(1): 3-11.

7. Mabvuure, N.T., Malahias, M., Hindocha, S., et al. Acute compartment syndrome of the limbs: current concepts and management. (2012) Open Orthop J 6: 535-543.

8. Giannoudis, P.V., Tzioupis, C., Pape, H.C. Early diagnosis of tibial compartment syndrome: continuous pressure measurement or not? (2009) Injury 40(4): 341-342.

9. Hargens, A.R., Akeson, W.H., Mubarak, S.J., et al. Fluid balance within the canine anterolateral compartment and its relationship to compartment syndromes. (1978) J Bone Joint Surg Am 60(4): 499-505. 10. Whitesides, T.E., Haney, T.C., Morimoto, K., et al. Tissue pressure measurements as a determinant for the need of fasciotomy. (1975) Clin Orthop Relat Res (113): 43-51.

11. McQueen, M.M., CourtBrown, C.M. Compartment monitoring in tibial fractures. The pressure threshold for decompression. (1996) J Bone Joint Surg Br 78(1): 99-104.

12. White, T.O., Howell, G.E.,Will, E.M., et al. Elevated intramuscular compartment pressures do not influence outcome after tibial fracture. (2003) J Trauma 55(6): 1133-1138.

13. Turnbull, D., Mills, G.H. Compartment syndrome associated with the Lloyd Davies position. Three case reports and review of the literature. (2001) Anaesthesia 56(10): 980-987.
14. Fleming, I, Egeler, C,. Regional anaesthesia for trauma: an update. (2013)

15. Turnbull, D, Farid, A., Hutchinson, S., et al. Calf compartment pressures in the Lloyd-Davies postion: a cause for concern? (2002) Anaesthesia 57(9): 905-908.

16. Tuckey, J. Bilateral compartment syndrome complicating prolonged lithotomy postion. (1996) Br J Anaesth 77(4): 546-549.

17. Malik, A.A., Khan, W.S., Chaudhry, A. et al. Acute compartment syndrome--a life and limb threatening surgical emergency. (2009) J Perioper Pract 19(5): 137-142.

18. Finkelstein, J.A., Hunter, G.A., Hu, R.W. Lower limb compartment syndrome: course after delayed fasciotomy. (1996) J Trauma 40(3): 342-324.

19. Fitzgerald, A.M., Gaston. P., Wilson, Y., et al. Long-term sequelae of fasciotomy wounds. (2000) Br J Plast Surg 53(8): 690-693.

20. Cheatham, M.L., Malbrain, M.L., Kirkpatrick, A., et al. Results from the International Conference of Experts on Intra-abdominal Hypertension and Abdominal Compartment Syndrome. II. Recommendations. (2007) Intensive Care Med 33(6): 951-962.

21. Luckianow, G.M., Ellis, M., Governale, D., et al. Abdominal compartment syndrome: risk factors, diagnosis, and current therapy. (2012) Crit Care Res Pract.

22. Lui, F., Sangosanya, A., Kaplan, L.J. Abdominal compartment syndrome: clinical aspects and monitoring. (2007) Crit Care Clin 23(3): 415-433.

23. Ridings, P.C., Bloomfield GL., Blocher, C.R., Blocher, C.R., et al. Cardiopulmonary effects of raised intra-abdominal pressure before and after intravascular volume expansion. (1995) J Trauma 39(6): 10711075 .

24. Maerz, L., Kaplan, L.J. Abdominal compartment syndrome. (2008) Crit Care Med 36(4 Suppl): S212-215.

25. Diaz, J.J. Jr., Cullinane, D.C., Dutton, W.D., et al. The management of the open abdomen in trauma and emergency general surgery: part 1-damage control. (2010) J Trauma 68(6): 1425-1438.
Ommega Online Publishers

Journal Title: Journal of Anesthesia and Surgery (JAS)

Journal Short Name: J Anesth Surg
Journal ISSN: 2377-1364

E-mail: anestheisa@ommegaonline.com

Website: www.ommegaonline.com 